\title{
Development and Testing of the Hayabusa2 Ion Engine System
}

\author{
By Kazutaka NishiYama, ${ }^{1)}$ Satoshi Hosoda, ${ }^{1)}$ Kazuma Ueno, ${ }^{1)}$ Ryudo TsukizaKI ${ }^{1)}$ and Hitoshi KuninakA ${ }^{1)}$ \\ ${ }^{1)}$ Institute of Space and Astronautical Science, JAXA, Sagamihara, Japan
}

(Received July 31st, 2015)

\begin{abstract}
Hayabusa2 is the second asteroid sample return mission by JAXA. The ion engine system (IES) for Hayabusa2 is based on that developed for Hayabusa with modifications necessary to improve durability, to increase thrust by $20 \%$, and to reflect on lessons learned from Hayabusa mission. Hayabusa2 will rendezvous with a near-earth asteroid 1999 JU3 and will take samples from its surfaces. More scientific instruments than Hayabusa including an impactor to make a crater and landers will be on board thanks to the thrust enhancement of the IES. An improved neutralizer with stronger magnetic field for longer life has been under endurance test in diode mode since August 2012 and has accumulated the operational hours of $25600 \mathrm{~h}$ (> mission requirement: $14000 \mathrm{~h}$ ) by July 2015. The IES flight model was developed within 2.5 years. The spacecraft was launched from Tanegashima Space Center in Kagoshima Prefecture on-board an H-IIA launch vehicle on December 3, 2014.
\end{abstract}

Key Words: Hayabusa2, Asteroid Explorer, Microwave Discharge, Ion Engine

\section{Introduction}

Japan's first asteroid explorer "Hayabusa" propelled by microwave discharge ion thrusters ${ }^{1)}$ came back to the earth on June 13, 2010. Hayabusa completed more than 7-year space mission, and finally Hayabusa could return the capsule to the earth, although Hayabusa had a lot of troubles and difficulties. ${ }^{2)}$ After careful inspections of about 1,500 grains found in the sample container, it turned out that we actually obtained rocky particles from the surface of Asteroid Itokawa. In 2011, Japan Aerospace Exploration Agency (JAXA) started the second project "Hayabusa2" for the asteroid sample return mission to another Asteroid 1999 JU3 which is one of different type (C-type) of asteroids from Itokawa (S-type). The Hayabusa2 flight system was designed based on the Hayabusa in order to make the development time as short as possible for the best launch window at the end of 2014. The critical design review (CDR) of the Hayabusa2 system was conducted in the beginning of FY2012, then flight model manufacturing and testing started. Figure 1 shows views of Hayabusa and Hayabusa2 spacecraft from the ion engine system (IES) side. Figure 2 illustrates trajectories of Hayabusa2 and 1999 JU3. Figure 3 depicts instruments on the spacecraft and component layout of the IES is completely the same as Hayabusa. Hayabusa2 was launched on December 3, 2014. It will arrive at the asteroid mid-2018 and return to Earth at the end of 2020.3) This paper briefly summarizes design, development, and ground testing of the IES for Hayabusa2.

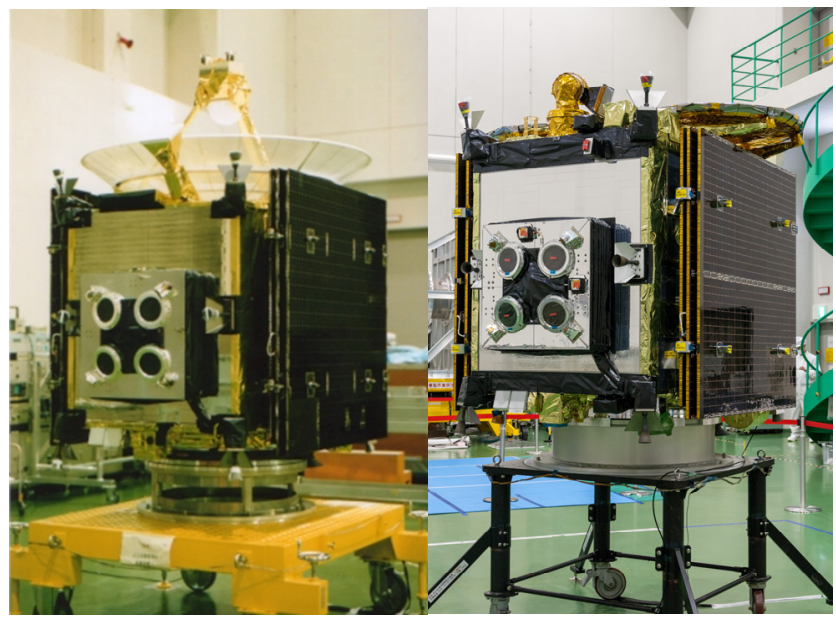

Fig. 1. Asteroid explorer Hayabusa launched in 2003 (Left) and its successor Hayabusa2 launched in 2014 (Right).

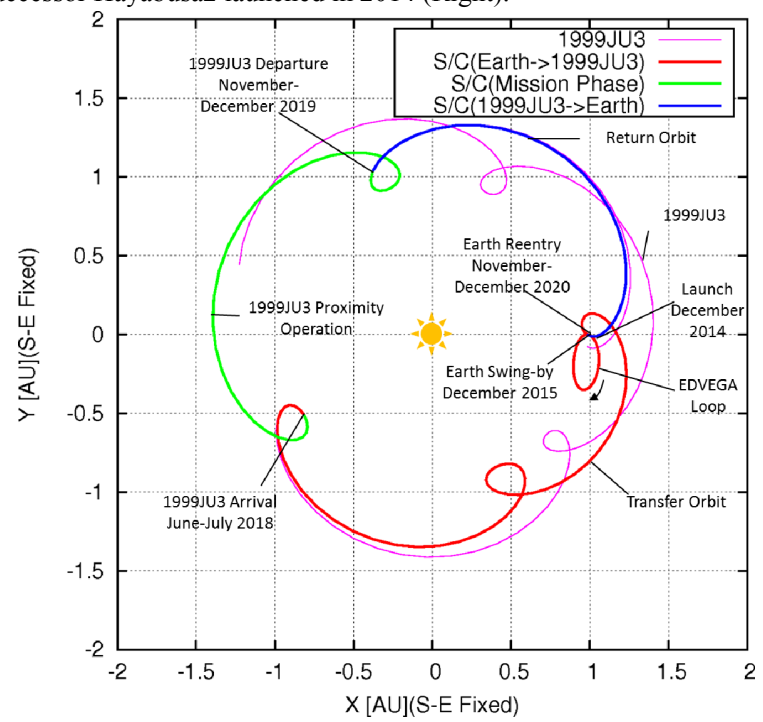

Fig. 2. Trajectories of Hayabusa2 and 1999 JU3 in the Sun-Earth fixed coordinate system; the Earth is located at $(1,0)$. 


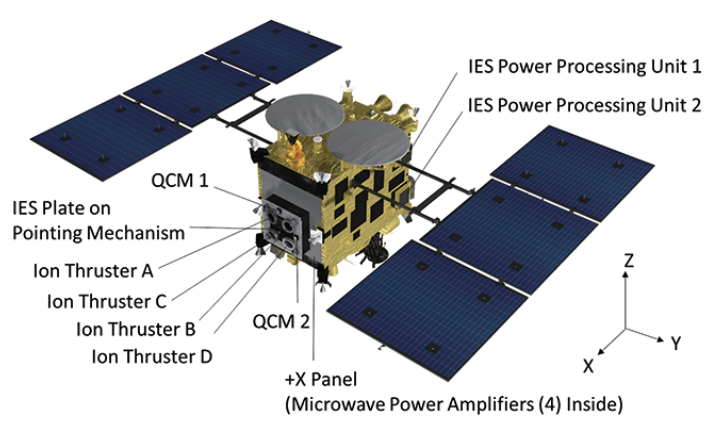

a)

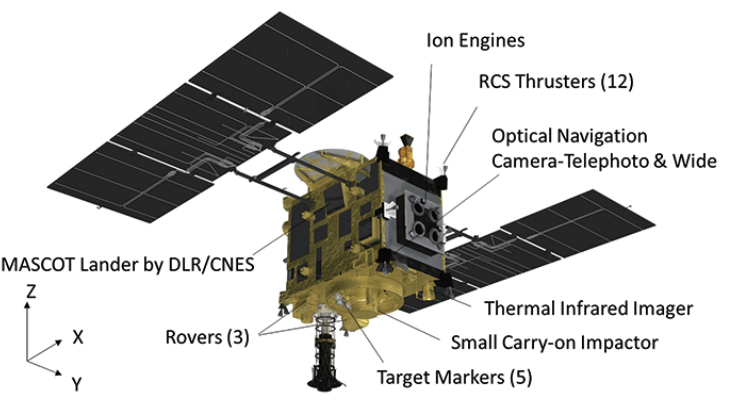

b)

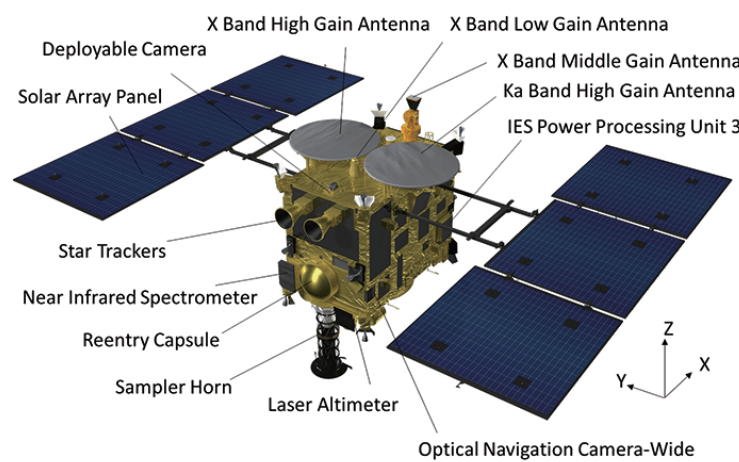

c)

Fig. 3. Hayabusa2 flight system. The dimension of the main body is $1 \mathrm{~m}$ $\times 1.6 \mathrm{~m} \times 1.25 \mathrm{~m}$.

\section{Microwave Discharge Ion Engine System}

This section summarizes the architecture, functions, mission requirements and development plan of the Hayabusa2's ion engine system.

\subsection{Hayabusa's Heritage and Post-Hayabusa Development Activities}

The ion engine system for Hayabusa2 has been developed based on that for Hayabusa. Both Hayabusa and Hayabusa2 have four $\mu 10$ ion thrusters on a single plate called "IES plate" which is mounted on top of a two-axis pointing gimbal mechanism as shown in Fig. 1 and 3. An ion thruster consists of an ion source and a neutralizer both of which utilize microwave discharge with electron cyclotron resonance at a frequency of $4.25 \mathrm{GHz}$. As for the original Hayabusa IES, the maximum thrust generated by a single thruster was $8 \mathrm{mN}$ by consuming a small flow rate (of order $0.3 \mathrm{mg} / \mathrm{s}$ ) of xenon gas and an electric power of $350 \mathrm{~W}$ for plasma generation and ion beam acceleration. A microwave generator is able to drive the ion source and the neutralizer by way of a coupler box
(CPBX) which divided microwaves in the ratio of four to one for the ion source and the neutralizer, respectively. Although the number of microwave power amplifiers is the same as the number of thrusters, there were only three IES power processing units (IPPUs) which contain three direct current power supplies for high-voltage beam acceleration and neutralizer current control. Three of four thrusters can be operated at the same time by selecting the dedicated IPPUs using three relay switching boxes (RLBX). Propellant xenon is supplied by a propellant management unit (PMU) which has a small accumulator downstream of a main tank by way of regulation valves for bang-bang pressurization. There are four ion thruster valves and eight flow restrictors for ion sources and neutralizers downstream of the accumulator. The xenon flow can only be shut off for each thruster but cannot be shut off individually for an ion source and a neutralizer. This limitation was similar to that of the microwave supply units because microwave power could not be stopped for either an ion source or a neutralizer, separately. Hayabusa's round-trip asteroid exploration was finally accomplished by these small microwave discharge ion engines. Total accumulated operational time reached 39637 hours for all four ion engines added up consuming $47 \mathrm{~kg}$ of xenon propellant. Total duration of powered spaceflight is 25590 hours which provided a delta-V of $2.2 \mathrm{~km} / \mathrm{s}$ and a total impulse of $1 \mathrm{MN} \cdot \mathrm{s}$, approximately, as summarized in the second column of Table 1 .

During the Hayabusa return trip since 2007, a joint activity of JAXA and NEC to generalize the IES for commercial satellites was started. In this program, many modifications were studied and implemented to a qualification model to make the IES more compatible and attractive to commercial geostationary small satellites of 1-1.5 tons class. Input voltage range of IPPU was expanded so that it can also be used on standard regulated bus voltages from 60 to $120 \mathrm{~V}$, as well as on original unregulated $100 \mathrm{~V}$. Ion thruster design was slightly changed and maximum thrust was increased by $20 \%$. The new IPPU is compatible with this higher beam current mode operation. New low-loss DC blocks were developed by ourselves instead of using foreign products. IES thruster control unit (ITCU) was enhanced so that it can control five thrusters at the same time (for possible thrust enhancement option for Hayabusa2 before the Preliminary Design Review), can accommodate two microwave oscillators for redundancy, can monitor subsystem temperatures independently from temperature control subsystem and can control two propellant management units as used in geostationary satellites. This joint development was supported by funding from both JAXA and NEC. NEC started collaboration with Aerojet to expand its low power microwave ion engine business in the U. S. market. These activities helped maintain our capabilities to deliver microwave ion engines again for Hayabusa2, 10 years after Hayabusa mission. These improved components became the baseline models for the Hayabusa2 IES and Hayabusa2 specific modifications were applied later. 


\subsection{IES Specifications and Mission Requirements}

Hayabusa2 IES specifications and mission requirements are summarized in Table 1. Ion sources were slightly modified in that their propellant injectors were added in different places in the discharge chamber and ion optics thicknesses and aperture diameters were changed so that the maximally available thrust increased by approximately $20 \%$ although specific impulse degraded by $10 \%{ }^{4)}$ The total impulse required by this new mission will be $1.2 \mathrm{MN} \cdot \mathrm{s}$ which is also $20 \%$ larger than Hayabusa's requirement. Estimated total operational hours of all thrusters are 41100 hours at maximum which is almost the same as Hayabusa achievement of 39637 hours. The increase of total impulse is due to the increase of spacecraft mass because required delta- $\mathrm{V}$ is almost the same as that of Hayabusa. This will be achieved by the thrust enhancement with almost the same thruster operational hours. The xenon load was nearly the same amount of $66 \mathrm{~kg}$ because both mission needs extra propellant for extended missions after return to the earth. Neutralizers were improved for longer life according to lessons learned in the Hayabusa mission and ground experiments. ${ }^{5)}$ Subsystem architecture of Hayabusa2 ion engines is almost identical to Hayabusa's although many components such as valves, pressure sensors, microwave components, and so on were discontinued in production and were replaced by new alternatives. These details will be described in the next section.

Table 1. IES specifications and mission requirements.

\begin{tabular}{|l|l|l|}
\hline & Hayabusa & Hayabusa2 \\
\hline Thrust per Thruster (mN) (MOL) & $4.4-7.6$ & $6.3-9.0$ \\
\hline Specific Impulse (s) (MOL) & $2760-3000$ & $2740-2890$ \\
\hline System Power (W) (MOL) & $280-1150$ & $380-1230$ \\
\hline Total Operational Hours (h·units) & 39637 & $<41100$ \\
\hline Powered Flight Duration (h) & 25590 & 13140 \\
\hline Total Impulse (MN·s) & 1.0 & 1.2 \\
\hline Total Delta-V (km/s) & 2.2 & 2.0 \\
\hline Dry Mass (kg) & 61 & 66 \\
\hline Xenon $(\mathrm{kg})$ & 66.2 & 66.5 \\
\hline Spacecraft Mass $(\mathrm{kg})$ & 510 & 608.6 \\
\hline
\end{tabular}

\section{Subsystem and Component Descriptions}

In this section, more details on the architecture, functions and design change of the components in the Hayabusa2 IES. Hierarchical structure and acronyms of ion engine system for Hayabusa 2 and interface block diagram are shown in Fig. 4 and 5, which are both identical to those of Hayabusa. IES mass and power breakdowns of Hayabusa2 and Hayabusa are shown in Table 2 and Table 3, respectively. Most of the components of Hayabusa2 are more massive than those of Hayabusa mainly due to replacement of discontinued foreign parts and partly due to the increase in maximum thrust level.

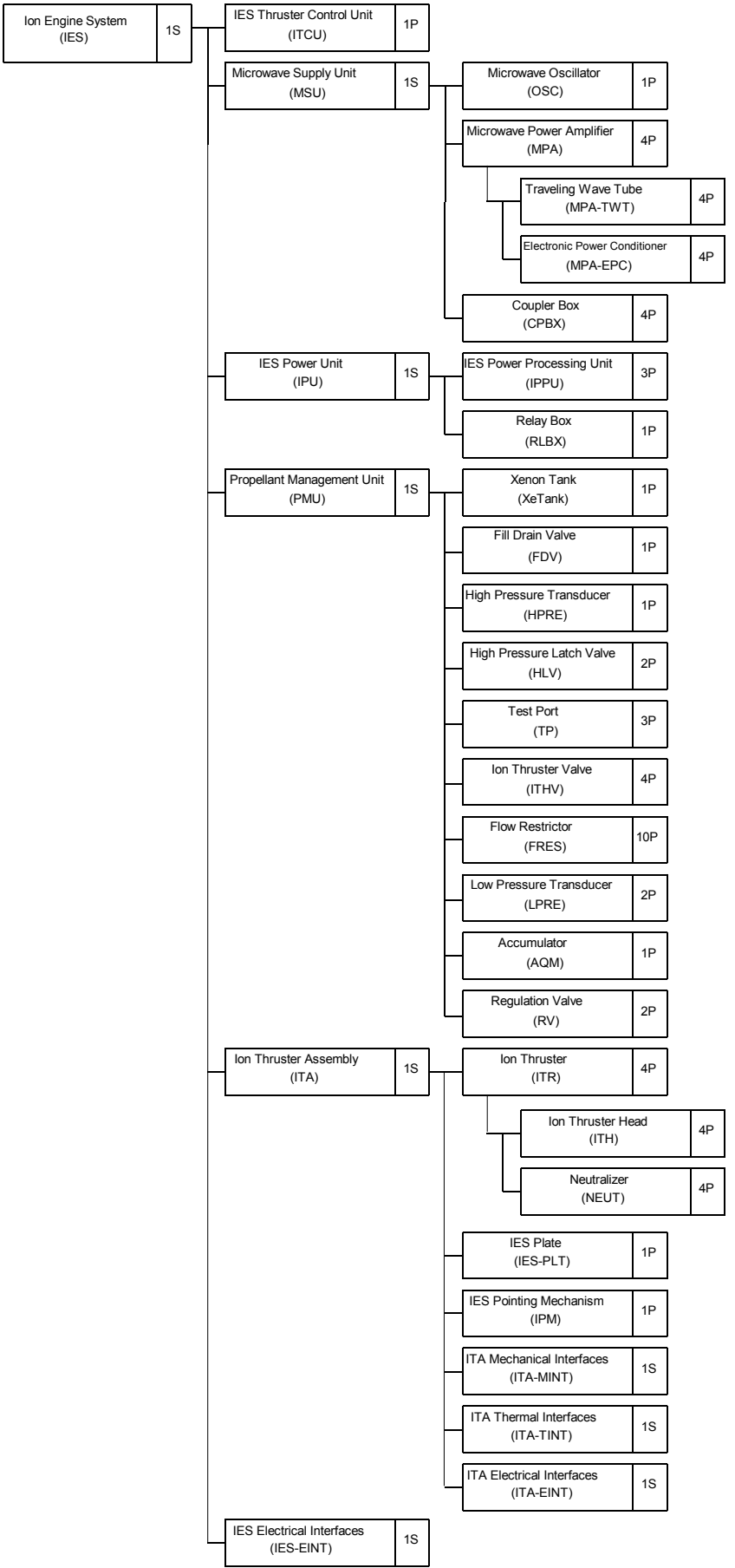

Fig. 4. Hierarchical structure and acronyms of ion engine system for Hayabusa2. 


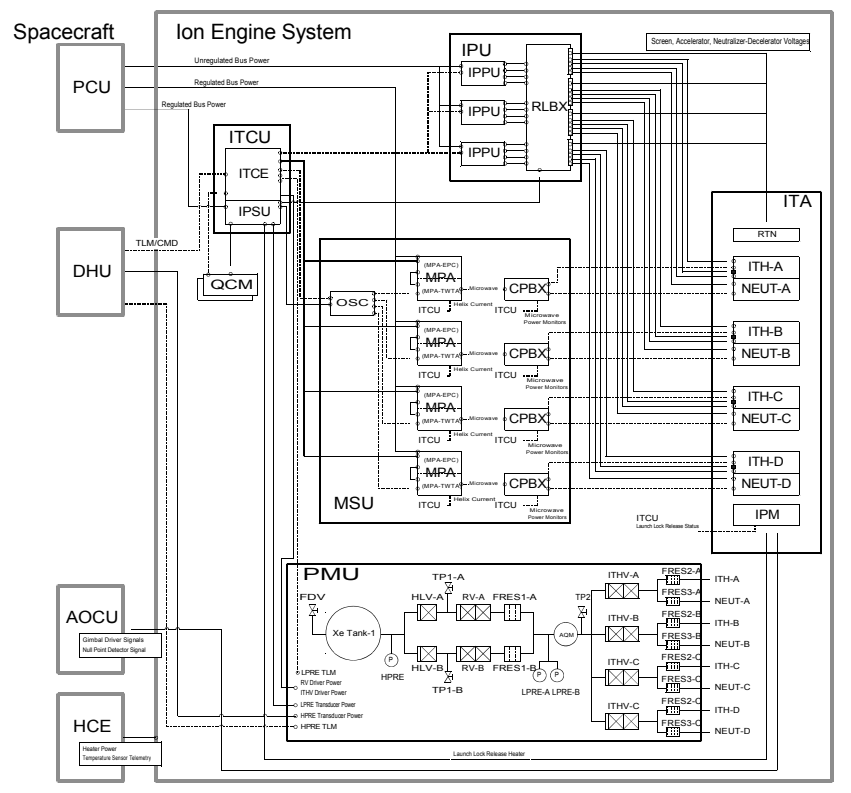

Fig. 5. Hierarchical structure and acronyms of ion engine system for Hayabusa2.

\begin{tabular}{|c|c|c|c|c|c|}
\hline Component & Acronym & QTY & $\begin{array}{l}\text { Unit } \\
\text { Mass } \\
(\mathrm{kg})\end{array}$ & $\begin{array}{l}\text { Total } \\
\text { Mass } \\
(\mathrm{kg})\end{array}$ & $\begin{array}{l}\text { Unit } \\
\text { Power (W) }\end{array}$ \\
\hline $\begin{array}{l}\text { IES Thruster } \\
\text { Control Unit }\end{array}$ & ITCU & 1 & 4.75 & 4.75 & $20-50$ \\
\hline $\begin{array}{l}\text { IES Power } \\
\text { Processing Unit }\end{array}$ & IPPU & 3 & 2.79 & 8.37 & $180-305$ \\
\hline Relay Box & RLBX & 1 & 1.06 & 1.06 & \\
\hline Oscillator & OSC & 1 & 0.27 & 0.27 & $(1.2)^{*}$ \\
\hline $\begin{array}{l}\text { Microwave } \\
\text { Power Amplifier }\end{array}$ & MPA & 4 & 2.24 & 8.96 & 103 \\
\hline Coupler Box & CPBX & 4 & 0.41 & 1.64 & \\
\hline $\begin{array}{l}\text { Propellant } \\
\text { Management Unit }\end{array}$ & PMU & 1 & 18.07 & 18.07 & $(4-30)^{*}$ \\
\hline $\begin{array}{l}\text { Ion Thruster } \\
\text { Assembly }\end{array}$ & ITA & 1 & 21.58 & 21.58 & \\
\hline $\begin{array}{l}\text { IES Electric } \\
\text { Interfaces } \\
\text { (High-Voltage } \\
\text { Harnesses, RF } \\
\text { Cables) }\end{array}$ & $\begin{array}{l}\text { IES } \\
\text { E-INT }\end{array}$ & lot & 1.67 & 1.67 & \\
\hline & & & Total & 66.37 & 314-1213 \\
\hline
\end{tabular}

${ }^{*}$ Included in ITCU power.
Table 3. Hayabusa IES components.

\begin{tabular}{|c|c|c|c|c|c|}
\hline Component & Acronym & QTY & $\begin{array}{l}\text { Unit } \\
\text { Mass } \\
(\mathrm{kg})\end{array}$ & $\begin{array}{l}\text { Total } \\
\text { Mass } \\
(\mathrm{kg})\end{array}$ & $\begin{array}{l}\text { Unit } \\
\text { Power (W) }\end{array}$ \\
\hline $\begin{array}{l}\text { IES Thruster } \\
\text { Control Unit }\end{array}$ & ITCU & 1 & 3.50 & 3.50 & $20-50$ \\
\hline $\begin{array}{l}\text { IES Power } \\
\text { Processing Unit }\end{array}$ & IPPU & 3 & 2.11 & 6.32 & $180-260$ \\
\hline Relay Box & RLBX & 1 & 0.77 & 0.77 & \\
\hline Oscillator & OSC & 1 & 0.27 & 0.27 & $(1.2)^{*}$ \\
\hline $\begin{array}{l}\text { Microwave Power } \\
\text { Amplifier }\end{array}$ & MPA & 4 & 2.28 & 9.11 & 103 \\
\hline Coupler Box & CPBX & 4 & 0.40 & 1.60 & \\
\hline $\begin{array}{l}\text { Propellant } \\
\text { Management Unit }\end{array}$ & PMU & 1 & 17.38 & 17.38 & $(4-30)^{*}$ \\
\hline $\begin{array}{l}\text { Ion Thruster } \\
\text { Assembly }\end{array}$ & ITA & 1 & 20.73 & 20.73 & \\
\hline $\begin{array}{l}\text { IES Electric } \\
\text { Interfaces } \\
\text { (High-Voltage } \\
\text { Harnesses, RF } \\
\text { Cables) }\end{array}$ & $\begin{array}{l}\text { IES } \\
\text { E-INT }\end{array}$ & lot & 1.34 & 1.34 & \\
\hline & & & Total & 61.02 & $314-1140$ \\
\hline
\end{tabular}

* Included in ITCU power.

\subsection{Ion Thruster Head}

The original well-tuned $\mu 10$ ion thruster can generate $8 \mathrm{mN}$ at a specific impulse (including neutralizer flow) of $3000 \mathrm{~s}$ with consuming $32 \mathrm{~W}$ of microwaves and $0.23 \mathrm{mg} / \mathrm{s}$ of xenon flow. Our work indicated that design change of gas injector layout had the large impact on thrust enhancement. ${ }^{4}$ The highest thrust of $10 \mathrm{mN}$ is generated when the xenon flow is divided at the ratio of 1:2 between the original injector deep in the waveguide and newly added injectors between magnets, respectively, when the total flow rate is $0.34 \mathrm{mg} / \mathrm{s}$. Mass utilization efficiencies are decreased from the original but can be improved by using a small hole accelerator grid whose aperture diameter was decreased from 1.8 to $1.5 \mathrm{~mm}$. Decrease of screen grid thickness from the original value of $0.95 \mathrm{~mm}$ to $0.8 \mathrm{~mm}$ also helped the mass utilization recovery. The combination of above mentioned modifications (gas distributors, small-hole accelerator grid and thinner screen grid) achieved the maximum thrust enhancement shown in Table 1 with acceptable decrease in specific impulse. This thrust enhancement was indispensable for Hayabusa2 mission because of the increase of spacecraft mass. The former laboratory experiments were conducted using two mass flow controllers to investigate the flow rate distribution ratio effects on thruster performance. To realize the optimum propellant feed balance in flight system as simply as possible, a gas flow divider was newly inserted between the gas isolator and the original gas port near the waveguide-end without changing design of the PMU. The divider consists of a small orifice connected to the waveguide and a branched gas pipe connected to the inter-magnet gas ports. Several orifice plates with different aperture diameters were manufactured and the ion source performance was tested whose result will be described in the later section. Because addition of the gas divider leaded to the upstream pressure rise which affected the gas isolator performance, the gas isolator design was also modified so that the number of stacked internal metal meshes and ceramics was increased. It was verified that the new gas 
isolator had a sufficiently high withstanding voltage at xenon flow rates lower than $0.5 \mathrm{mg} / \mathrm{s}$. Material of the support ring of ion optics was changed from a discontinued Al-Si alloy to a metal matrix composites of $\mathrm{SiC}$ and $\mathrm{Al}$. By this change, thermal expansion coefficient of the ring material decreased from $16 \times 10^{-6} \mathrm{~K}^{-1}$ to $7 \times 10^{-6} \mathrm{~K}^{-1}$ that contributes to decrease thermal grid-gap change and to relax thermal stress of the carbon-carbon composite grids. A microwave feedthrough made in USA (an antenna with a TNC coaxial connector) was replaced with a newly developed domestic one for better availability.

\subsection{Neutralizer (NEUT)}

Lifetimes of Hayabusa neutralizers were ranges between 9579 and 14830 hours which are both much shorter than the ground test's achievement of 20000 hours by a prototype model (voluntarily stopped). Number of on/off cycles does not seem to be dominant because the longest life was achieved by the one most frequently switched on and off. Most remarkable difference between in-space and on-ground would be the temperature range. Hayabusa ion engines experienced extremely low temperatures several times by accidents and it might have somehow shorten the life time of neutralizers. We have analyzed the stored prototype neutralizer by disassembling it and found many magnetized metal flakes stuck on magnetic yokes where local magnetic surface flux densities are large. These metal flakes may be immersed into the discharge plasma, may become a new source of surface contamination of the dielectric part of microwave launcher by ion sputtering and sputtered metal deposition, and may increase plasma loss. All the flight and ground test data have a common feature that there is a synchronized increase of neutralizer coupling voltage and neutralizer backward power. This may be caused by above mentioned metal coating of dielectric surfaces of a microwave launcher. A start point of the synchronized increases would correspond to an occurrence of delamination and sticking of a piece of flake. Improvement of the neutralizer reliability was the most important work of the Hayabusa2 IES, and design of the magnetic field was improved by increasing the number of SmCo magnets and changing the magnetic yoke shape. The new neutralizers have $20 \%$ stronger magnetic field for better plasma confinement. About 500 hours of diode mode operations of a laboratory model of neutralizer with different magnetic field strengths indicated us that the stronger magnetic field decreased the erosion or deposition rates of metallic parts used in the discharge chamber.

Another improvement is about neutralizer bracket's electrical insulation. Leakage currents were observed through an electric short circuit at the neutralizer's insulator surfaces contaminated by deposition of sputtered metallic materials generated during crossed operation of the neutralizer $\mathrm{A}$ and the ion source B in the final approach to Earth by Hayabusa in which the spacecraft was highly negatively charged relative to the ion engine plume plasma. We improved shadow shielding of the dielectric insulator between the neutralizer bracket and the IES plate. A microwave feedthrough made in USA (an antenna with an SMA coaxial connector) was replaced with a newly developed domestic one for better availability.
During the Hayabusa2 IES development, the neutralizer was the only component that required production of engineering models to conduct qualification tests including long term endurance test in diode mode operation.

\subsection{IES Power Processing Unit (IPPU)}

The IPPU converts the $60-120 \mathrm{~V}$ input power from spacecraft solar arrays (unregulated power bus) into the currents and voltages needed by thruster operation. The IPPU is almost the same as the Hayabusa IPPU with the following exceptions:

1. One of three Hayabusa IPPUs showed unstable oscillatory current limiting behavior at high temperatures in the first year in space. This has already been fixed in the qualification model development for the "generalized" IES.

2. Higher beam current operation up to $180 \mathrm{~mA}$ is possible and qualified.

3. Options for constant current operation target of neutralizer current were changed. Original IPPUs had three options: $\times 1$ of the corresponding screen current (nominal operation), $\times 1.5$ (two neutralizers for three ion sources on one neutralizer failure) and $\times 2$ (one neutralizer for two ion sources on one neutralizer failure). We will have different three options for Hayabusa2: NOMINAL (screen current + $3 \mathrm{~mA}$ ), HIGH (screen current $+10 \mathrm{~mA}$ ) and OFF (the neutralizer will be fixed to the spacecraft potential without negative bias voltage).

4. The high voltage output connector was replaced with another type due to availability. The mass increase of the IPPU was partly caused by this.

\subsection{Propellant Management Unit (PMU)}

The PMU feeds xenon gas to ion sources and neutralizers. Flow rates for higher thrust operation are a little larger than in the nominal thrust operation of Hayabusa IES. However, no design change is required about this. Only the design change will be replacement of discontinued solenoid valves for regulation valves (RV) and thruster valves (ITHV) to different space-qualified models provided by Moog which were also used in $\mathrm{DS}^{6}{ }^{6}$ and $\mathrm{Dawn}^{7}$. The low pressure transducer (LPRE) of Hayabusa showed noisy output when the IPPUs were switched on, which degraded flow rate (or thrust level) control accuracy. These transducers were replaced with different models which were equipped with internal current limiters according to JAXA's standard, and the electromagnetic interference as mentioned before was not observed any longer.

In the Hayabusa2 project sinusoidal vibration testing was required which were not applied in the format Hayabusa project because of the change of launch vehicle from the Japan's M-V solid rocket to the H-IIA liquid propellant rocket. To cope with this change four xenon tank brackets were slightly modified but mass change due to this was negligible. Mass gain of the PMU is mainly due to the change of solenoid valves.

\subsection{Microwave Supply Unit (MSU)}

The MSU is for ECR plasma generation and includes traveling wave tube amplifiers (TWTA) and passive 
microwave components such as flexible cables, semi-rigid cables, coupler boxes (CPBX) for microwave distribution between an ion source and a neutralizer, and DC blocks. Several parts such as microwave receptacles and detectors used in the CPBX were replaced with alternatives due to availability, but there was essentially no design change. TWTAs were replaced by successors of Hayabusa models of the same manufacturer. The preheat time for the TWT cathode was changed from $180 \mathrm{~s}$ to $200 \mathrm{~s}$ that was acceptable because this time interval was a variable in the control logic of the IES thruster control unit (ITCU). The vender of flexible coaxial cables were switched and unified for entire spacecraft including IES and communication subsystem in accordance with JAXA's space electronic parts selection standard. Figure 6 shows pictures of new low-loss DC blocks jointly developed by JAXA and NEC. DC Blocks are coaxial components that prevent the application of high-voltage direct current (DC) for the ion sources to TWTAs and CPBXs while offering minimum interference to RF signals. They have capacitors (insulation films) in series with both the inner and outer conductors. The new one has an insertion loss of $0.2 \mathrm{~dB}$ which is $0.3 \mathrm{~dB}$ smaller than the Hayabusa's made in USA. This slightly improve electrical efficiency of the thruster and reduce heat dissipation. The oscillator was replaced by the one newly developed by NEC due to non-availability of the original parts. The new oscillator has a built-in power divider for four outputs.

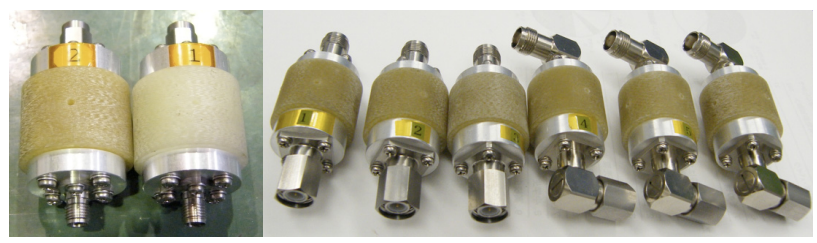

Fig. 6. Newly developed low-loss DC blocks for neutralizers (Left with SMA connector) and ion sources (Right with TNC connector).

\subsection{IES Thruster Control Unit (ITCU)}

The ITCU is the controller of the IES. Details of its function were described in a reference. ${ }^{8)}$ In the generalization and commercialization program, a qualification model of enhanced ITCU was developed. However, most of the enhancement will be removed and lightweight for Hayabusa2. Only the new capability will be the generation of user packet telemetry that contains accumulated impulse (time integration of the thrust calculated from voltages and currents). This compact telemetry data will be quickly downloaded and used for radiometric orbit determination without downloading much larger spacecraft housekeeping data which also contains the same telemetry data. This improvement is based on lessons learned from seven-year Hayabusa mission.

Another improvement is about the xenon flow rate control. The original ITCU for Hayabusa controlled the accumulator tank pressure by open and close the regulation valve (RV) referring the low pressure transducers (LPRE) output, which we called "delta-P control". Pressure (or flow rate) ripple was relatively large because of the lack of pressure telemetry's resolution and slow response due to the ITCU's internal analog filter whose time constant was about $1 \mathrm{~s}$. As a result, minimum valve opening duration was limited to $2 \mathrm{~s}$ and pressure ripple was twice as large as we expected. Now the new ITCU has another control method "delta-T control" in which valve opening duration can be set as a multiplier of the minimum value $62.5 \mathrm{~ms}$. At the initial main tank pressure of 7 $\mathrm{MPa}$ after launch, the valve opening duration of $500 \mathrm{~ms}$ is the nominal setting for cruise operation.

\subsection{IES Pointing Mechanism (IPM)}

The IPM consists of two-axis gimbals and launch lock mechanisms. Electrical harnesses for wax motors and hall sensors were improved for better stress relief and electromagnetic compatibility. In order to comply with the sinusoidal vibration specifications, some parts were slightly improved in its mechanical strength by increasing thickness of the supporting legs and number of fasteners and by adding shear pins. Mass increase by these improvement was only 32 grams $(+1 \%$ of the original).

\subsection{Ion Thruster Assembly (ITA)}

Hayabusa2's ion thruster cant angles were slightly changed from those of Hayabusa because relative position to the spacecraft center of mass was different from the Hayabusa. The thickness of the IES plate was also changed so that the center of mass of the plate and four thrusters is close to the gimbal's pivot point because the thruster's mass distribution was different due to design changes for the thrust enhancement or the material change as already mentioned. Two quarts crystal microbalances (QCM) made in Japan were installed on the outer surface of the ion engine plate as depicted in Fig. 3a in order to monitor surface erosion or deposition which might be caused by ion thruster operation. Those locations are similar to but not exactly the same as those of solar cell contamination monitors of Hayabusa IES. ${ }^{9)}$ The QCMs were the same lot as the one that has already been space qualified in the JAXA's Small Demonstration Satellite-4 (SDS-4) satellite mission and has been operating for three years in a low earth orbit. ${ }^{10)}$

\section{Flight Model Development and Ground Testing}

\subsection{Ion thruster head development}

The ion thruster head (or ion source) has a small-hole accelerator grid in order to improve the neutral atoms confinement in the discharge chamber. The newly assembled ion optics indicates severe direct impingement of the ion beams on the accelerator grid. To avoid possible internal contamination of the discharge chamber wall of the FM ITH by the carbon atoms sputtered out of the grid, a qualification model (QM) thruster which was designed completely the same as the FM was used for "grid aging". FM grid assemblies were installed to the QM thruster's discharge chamber and operated for about 10 hours one after the other. Accelerator grid currents were initially $3 \mathrm{~mA}$ at a screen current of $160 \mathrm{~mA}$ and gradually decreased to 1-1.5 mA. These grids experienced 2000-5000 times of high voltage breakdowns during these aging process. Surfaces between screen and accel. grids were polished with sandpaper several times to remove the carbon deposition generated by breakdowns or ion sputtering. 
Lengths of the waveguide and the antenna, and the diameter of gas divider are the performance tuning parameters of the ion thruster head. Ion thrusters of Hayabusa had different geometric parameters as a result of cut and try of each thruster. However, we decided that the common parameters should be applied to all four FM thrusters of Hayabusa2 and parameter survey should be completed using QM thruster in advance. The best combination of these parameters to achieve the required maximum screen current of $170 \mathrm{~mA}$ at a sufficiently high specific impulse were finally determined. Plasma ignition easiness is also the important requirement for the ion source. According to our experiments, larger flow rate requires larger microwave power for ignition. The worst case plasma ignition test was conducted by the following procedure. The xenon gas was fed to the QM thruster at a mass flow rate of $0.49 \mathrm{mg} / \mathrm{s}$ which is much larger than nominal flow rate of $0.31 \mathrm{mg} / \mathrm{s}$ for the maximum thrust operation, and then the nominal microwave power of $32 \mathrm{~W}$ was launched into the discharge chamber. When the best parameters were applied, xenon plasma was instantly generated. However, if the antenna length was longer than a threshold length, plasma ignition was possible only at smaller mass flow rates. The selected antenna length for FM thrusters is sufficiently shorter than this critical length. The best geometric parameters found in the QM thruster experiments were applied to all the four FM thrusters. After assembly of the FM thrusters, 100-h continuous operations were conducted for thruster aging. Figure 7 shows luminosities of the ion source and the neutralizer of the FM thruster B in a vacuum chamber. Figure 8 shows the screen current as a function of mass flow rate for different orifice diameters of gas divider for the FM thruster C. Effect of the orifice diameter was the same as in the case of the QM thruster. Thus the similar test using other three FM thrusters were skipped. A larger orifice increases xenon flow rate fraction to the waveguide gas port compared to the inter-magnet gas port. A screen current of $170 \mathrm{~mA}$ was the development target that was achieved with the $0.5-\mathrm{mm}$ diameter orifice at the smallest mass flow rate (i.e., at the highest specific impulse).

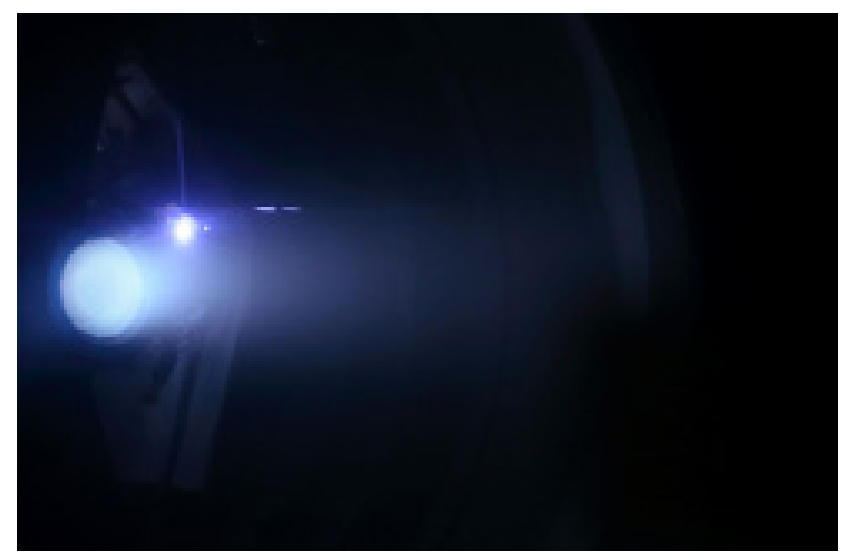

Fig. 7. Ground operation of the FM thruster B.

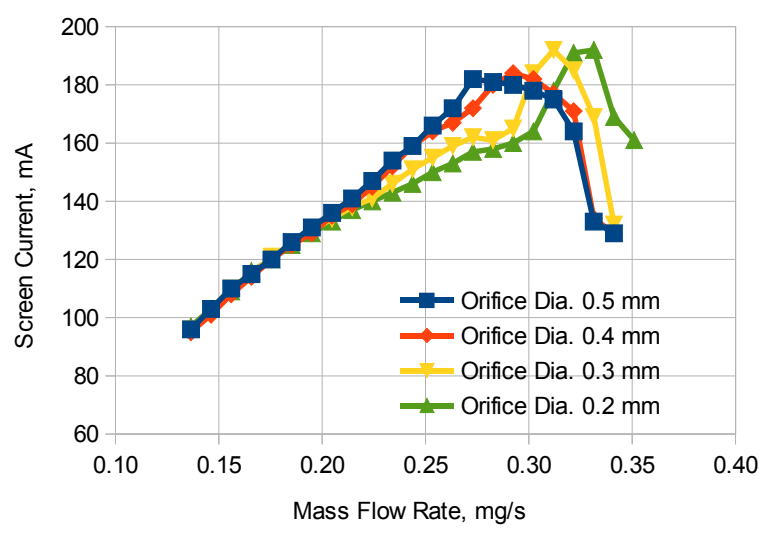

Fig. 8. Throttling curves of the FM ion source $\mathrm{C}$ for different orifice diameters of the gas divider.

\subsection{Neutralizer development}

Minimization of the operating voltage to improve the neutralizer durability were achieved by increasing the magnetic field inside the discharge chamber and by more intensive microwave impedance matching effort using a single stub tuner. Lengths and microwave phase angles of microwave coaxial cables for neutralizers were common for all four neutralizers and severely controlled although these parameters were not unified in the old Hayabusa IES. As of July 2015, still continuing endurance test in diode mode operation of an engineering model of new neutralizer, including once-per-week on-off cycles like in orbit cruise operation, accumulated the operational hours of $25600 \mathrm{~h}$ without any performance degradations. Operating temperature was controlled to $175^{\circ} \mathrm{C}$ by heaters attached on the neutralizer and cooled down to $5-10{ }^{\circ} \mathrm{C}$ once a week by a cold air flow through a cooling pipe around the neutralizer. Figure 9 shows the progress of the endurance test since August 2012. Figure $9 \mathrm{~b}$ shows initial anomaly of the neutralizer voltage when the voltage reached $47 \mathrm{~V}$. This was caused by an accidental heater malfunction, outgassing and contamination of a punching metal anode surface, which was soon recovered. Until $4000 \mathrm{~h}$ have passed, the neutralizer voltage changed unstably. It seems that air exposures affects the neutralizer voltage. Since $4000 \mathrm{~h}$ neutralizer has been kept in vacuum or in nitrogen gas when it stopped operation every week or several times per year for planned blackout. Thanks to this care, the voltage became quite stable since then. The evolution of the flow-rate throttling characteristics of the neutralizer is shown in Figure 10 which are also stable after $4000 \mathrm{~h}$. This endurance test will be continued as long as possible although the mission requirement of $14000 \mathrm{~h}$ has already passed. 


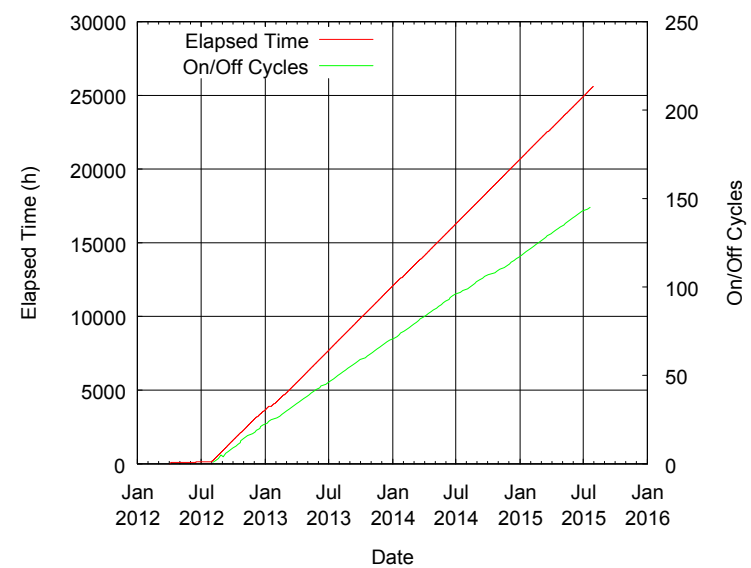

a)

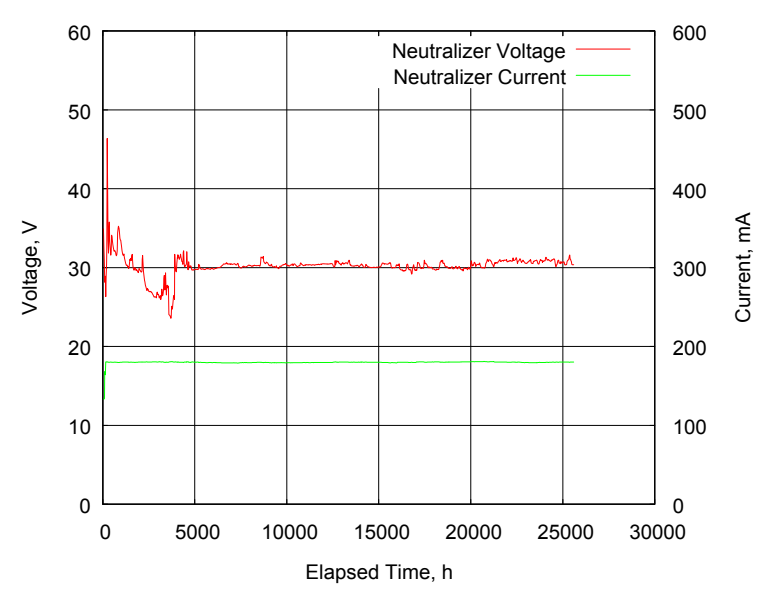

Fig. 9. Progress of an endurance test of the EM neutralizer.

a) Accumulated operating hours and on/off cycles. The neutralizer is switched off and cooled down once a week to simulate weekly flight operations. b) Daily average history of neutralizer voltage and current at a microwave power of $8 \mathrm{~W}$ and a xenon mass flow rate of $68 \mu \mathrm{g} / \mathrm{s}$.

a)

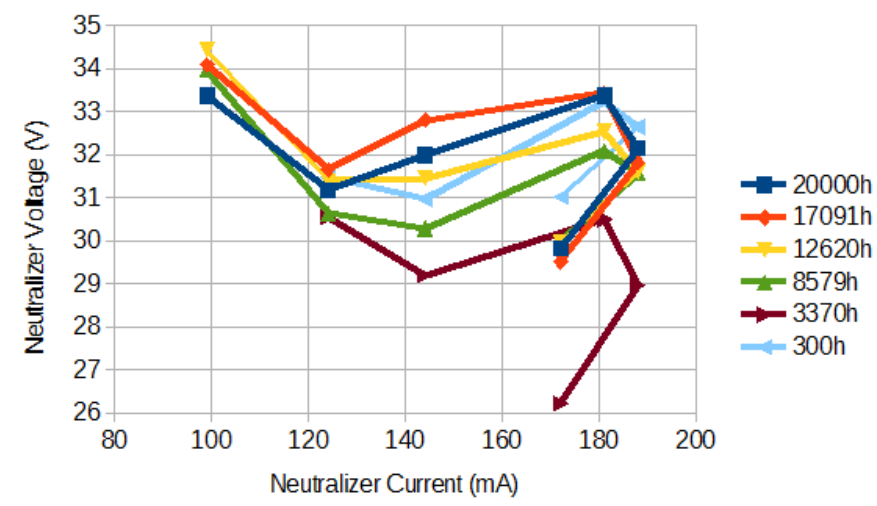

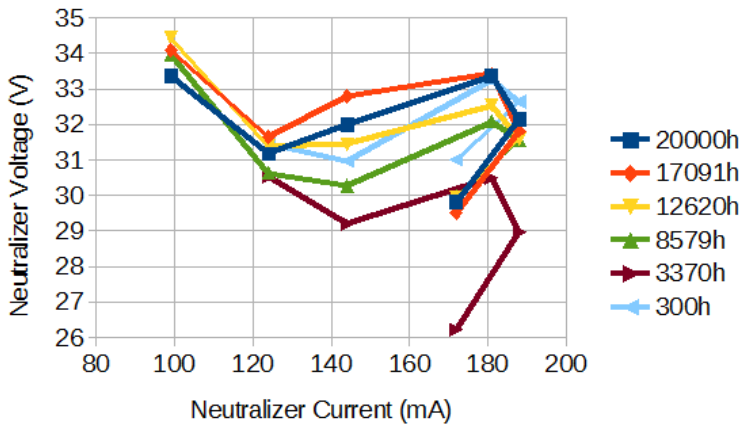

a)

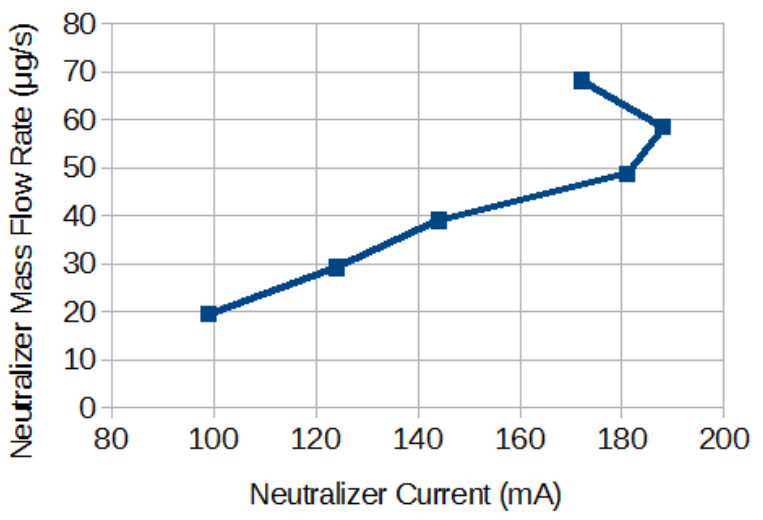

b)

Fig. 10. Time evolution of the EM neutralizer performance.

a) V-I curves of the EM neutralizer for different elapsed times during the endurance test. b) Simulated neutralizer current throttling in accordance with the mass flow rate. This curve is determined from the beam current dependency on the flow rate of the QM ion source. The neutralizer flow rate is controlled proportionally to that of the ion source.

\subsection{Subsystem integration test}

Subsystem integration test using flight models of ITR, MSU, IPPU, and ITCU was conducted in March and April 2015. Xenon gas was supplied using commercial mass flow controllers instead of using the FM-PMU. Microwave cables were substituted by test cables with the same lengths and insertion losses of flight cables. Figure 11 shows the throttling curves for the screen current and the neutralizer voltage for all the thrusters. Nominal thruster operation in space will be conducted at ion source mass flow rates smaller than 0.32 $\mathrm{mg} / \mathrm{s}$. These performances were affected by the characteristics of microwave coupler boxes (CPBX). Because CPBX's microwave power division ratio between the ion source and the neutralizer is sensitive to the intensity and the phase of the reflected microwaves from the ion source, end-to-end testing of the MSU and the ITR is important. As shown in the Fig.11b, neutralizer voltage never exceeded $35 \mathrm{~V}$ at flow rates lower than $0.32 \mathrm{mg} / \mathrm{s}$, which was one of the development goals. 


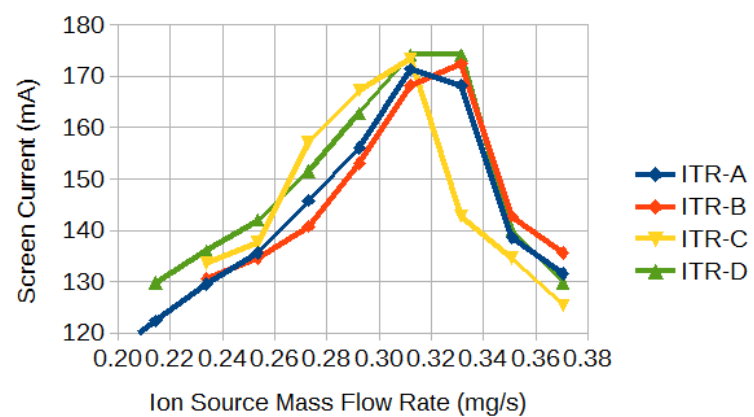

a)

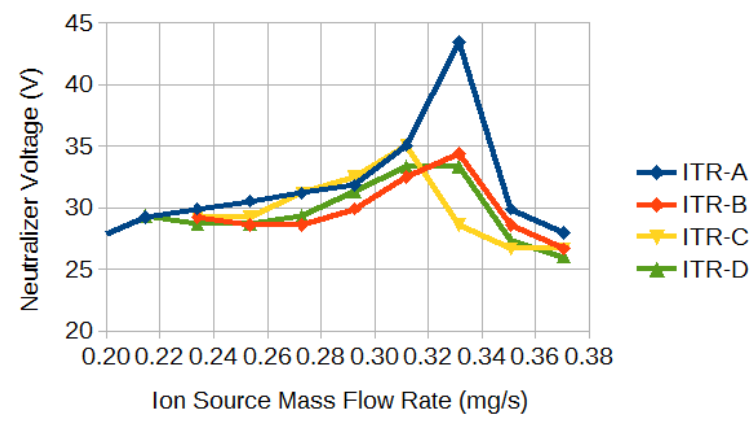

b)

Fig. 11. Result of the subsystem integration test using FM-ITR, FM-MSU, FM-IPPU, and FM-ITCU.

a) Screen currents of the FM ion thrusters. b)Neutralizer voltages of the FM ion thrusters.

\subsection{Spacecraft system integration}

The IES was integrated into the Hayabusa2 system in May 2014. After the system's mechanical environment tests, the spacecraft was installed into a large vacuum chamber for the thermal vacuum test (TVT) as shown in pictures in Fig. 12. In the system TVT, two or three ion thrusters were operated at a time in "discharge-only-mode" without ion beam extraction. In order to evaluate the heat dissipation from ITR, MSU, IPPU of the IES without beam extraction, dummy loads were located outside the vacuum tank and high voltages from IPPUs were supplied to them.

After the TVT finished, the IES firing test was carried out in June 2014. A beam target was attached to the spacecraft support and the IES plate and other spacecraft surfaces were covered with masking films to avoid contamination by the sputtered atoms from the target. The main purpose of this test was to confirm the workmanship on the thruster insulations when the IES was installed into the spacecraft system. Firstly, generation of open circuit voltages by IPPUs were tested. In some cases, high voltage breakdowns at ion optics were observed by a CCD monitoring camera but finally high voltages were stably applied to the thrusters. Then ion beam acceleration of one thruster at a time was tried. Though frequent high voltage breakdowns at grid gap limited the duration of the continuous beam extraction to 1-20 $\mathrm{s}$, generation of plumes of ion beam and neutralizer plasma was recorded by the CCD camera as shown in Fig. 13. The purpose of this test was not to demonstrate steady state ion beam extraction in the poor vacuum environment such as high background pressure and severe back sputtering from the close target, but to confirm that there was not electrical misconnection or dead short. Spacecraft telemetry data on the IES were reasonable and the firing test was successfully finished.

a)

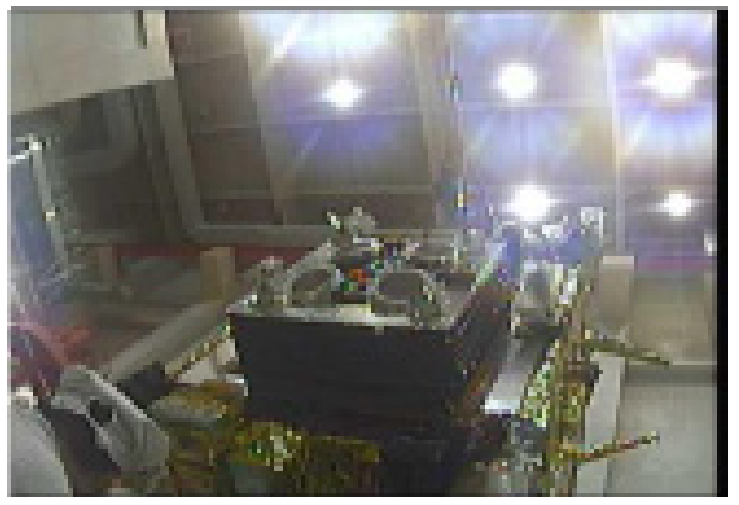

b)

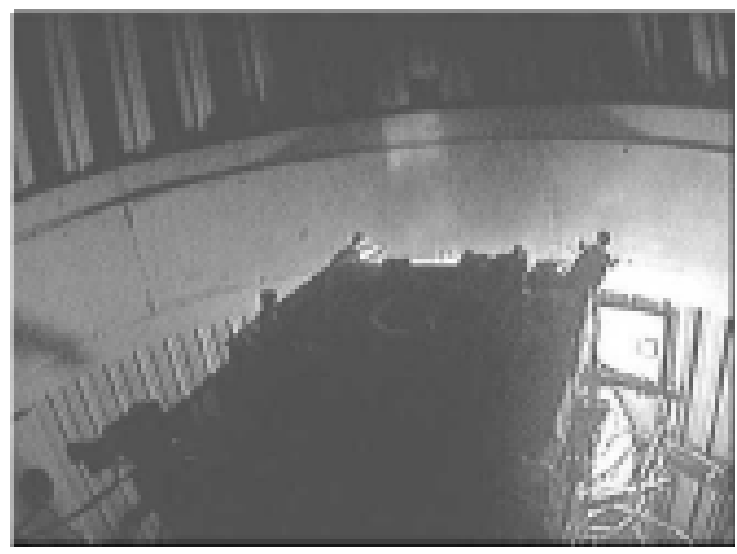

c)

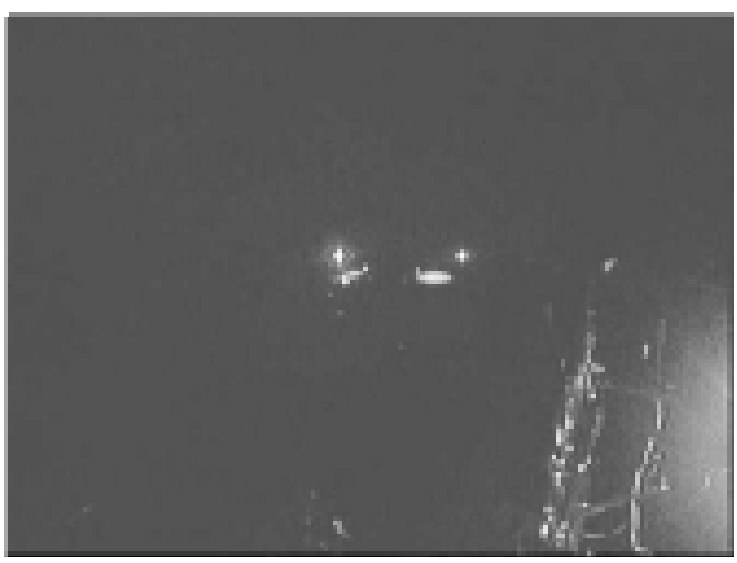

Fig. 12. Discharge-only operation of the ion engine system during the thermal vacuum test of the spacecraft.

a) Spacecraft outside the vacuum chamber. b) Spacecraft installation to the vacuum chamber. c)Plasmas of thruster A and B were generated. 
a)

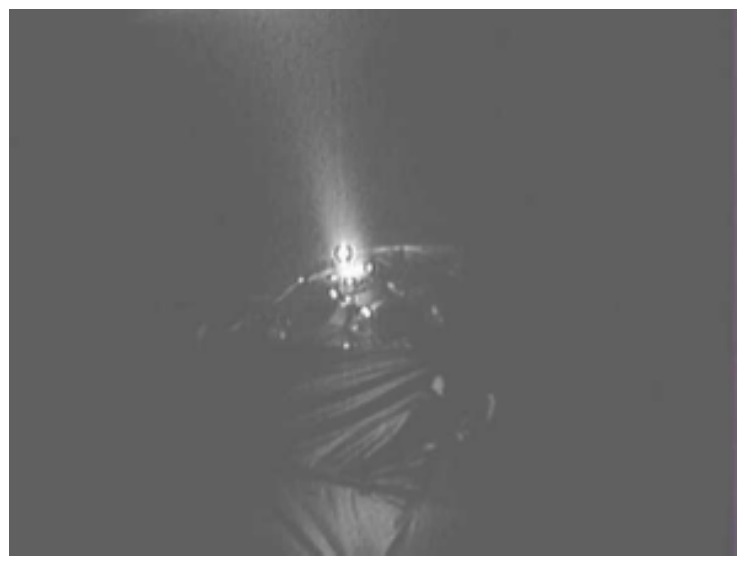

b)

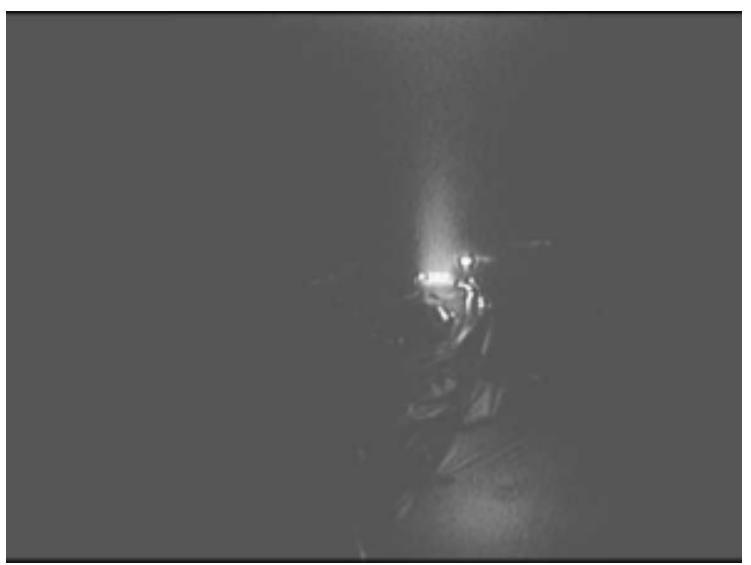

c)

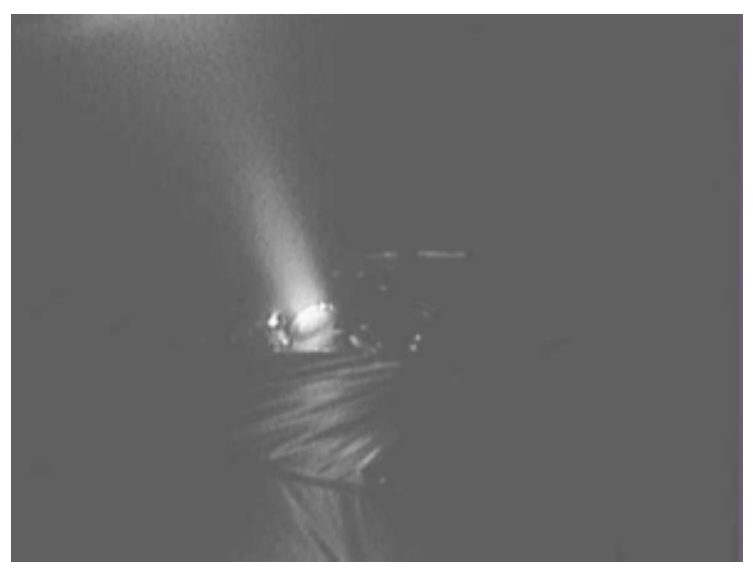

d)

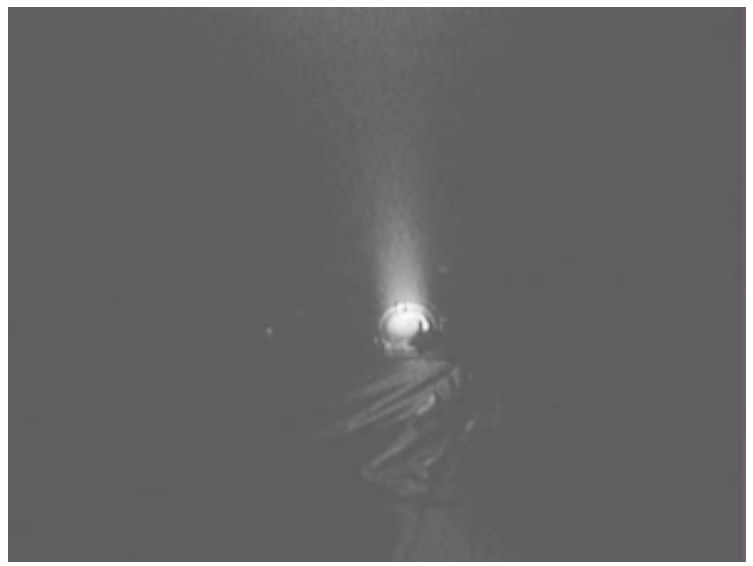

Fig. 13. End-to-end firing test of ion thrusters on the spacecraft in a vacuum chamber.

a) Thruster A. b) Thruster B. c) Thruster C. d) Thruster D.

\section{Conclusion}

The flight model of Hayabusa2 ion engine system was developed within 2.5 years, which was very challenging for authors. The spacecraft was launched from Tanegashima Space Center in Kagoshima Prefecture on-board an H-IIA launch vehicle on December 3, 2014. Initial check out of the IES in orbit was successfully completed by the end of February 2015 and powered flight for Electric Delta-V Earth Gravity Assist (EDVEGA) started in March 2015. ${ }^{11)}$

\section{References}

1) Kuninaka, H.: Development and Demonstration of a Cathodeless Electron Cyclotron Resonance Ion Thruster, Journal of Propulsion and Power, 14, No. 6 (1998), pp. 1022-1026.

2) Nishiyama, K., Hosoda S., Koizumi, H., Shimizu, Y., Funaki, I., Kuninaka, H., Bodendorfer, M. and Kawaguchi, J.: Hayabusa's Way Back to Earth by Microwave Discharge Ion Engines, 46th AIAA/ASME/SAE/ASEE Joint Propulsion Conference \& Exhibit, AIAA-2010-6862, 2010.

3) Kuninaka, H. and Hayabusa-2 Project: Deep Space Exploration of Hayabusa-2 Spacecraft, 30th International Symposium on Space Technology and Science, 2015-k-61, 2015.

4) Nishiyama, K., Hosoda S., Koizumi, H., Shimizu, Y., Kuninaka, H. and Tukizaki, R.: Feasibility Study on Performance Enhancement Options for the ECR Ion Thruster $\mu 10$, 44th AIAA/ASME/SAE/ASEE Joint Propulsion Conference \& Exhibit, AIAA-2008-4539, 2008.

5) Ohmichi, W. and Kuninaka, H.: Performance Degradation of a Spacecraft Electron Cyclotron Resonance Neutralizer and Its Mitigation, Journal of Propulsion and Power, 30, No. 5 (2014), pp. 1368-1372.

6) Brophy, J. R. et al.: Ion Propulsion System (NSTAR) DS1 Technology Validation Report, JPL Publication No. 00-10, 2000.

7) Brophy, J. R. et al.: Development and Testing of the Dawn Ion Propulsion System," 42nd AIAA/ASME/SAE/ASEE Joint Propulsion Conference \& Exhibit, AIAA 2006-4319, 2006.

8) Nishiyama, K., Funaki, I., Kuninaka, H. and Toki, K.: Development of an Ion Thruster Control Unit for the MUSES-C Mission, 26th International Electric Propulsion Conference, IEPC 99-065, 1999.

9) Nishiyama, K. and Kuninaka, H.: Development of Spacecraft Surface Contamination Sensors, International Astronautical Congress, IAC-09.C2.6.5, 2009.

10) Nishiyama, K. and Kuninaka, H.: Development and Flight Experiment of a Space QCM in Small Demonstration Satellite-4, Trans. JSASS Aerospace Tech. Japan, 12, No. ists29 (2014), pp.Tr_19-Tr_25.

11) Hosoda, S., Nishiyama, K., Tsukizaki, R. and Kuninaka, H.: Initial Checkout after Launch of Hayabusa2 Ion Engine System, 30th International Symposium on Space Technology and Science, 2015-b/IEPC-334, 2015. 